\title{
Predicting synthetic lethal genetic interactions in Saccharomyces cerevisiae using short polypeptide clusters
}

\author{
Yuehua Zhang ${ }^{1}$, Bo Li ${ }^{1}$, Pradip K Srimani ${ }^{1}$, Xuewen Chen ${ }^{2}$, Feng Luo ${ }^{1 *}$ \\ From IEEE International Conference on Bioinformatics and Biomedicine 2011 \\ Atlanta, GA, USA. 12-15 November 2011
}

\begin{abstract}
Background: Protein synthetic lethal genetic interactions are useful to define functional relationships between proteins and pathways. However, the molecular mechanism of synthetic lethal genetic interactions remains unclear.

Results: In this study we used the clusters of short polypeptide sequences, which are typically shorter than the classically defined protein domains, to characterize the functionalities of proteins. We developed a framework to identify significant short polypeptide clusters from yeast protein sequences, and then used these short polypeptide clusters as features to predict yeast synthetic lethal genetic interactions. The short polypeptide clusters based approach provides much higher coverage for predicting yeast synthetic lethal genetic interactions. Evaluation using experimental data sets showed that the short polypeptide clusters based approach is superior to the previous protein domain based one.

Conclusion: We were able to achieve higher performance in yeast synthetic lethal genetic interactions prediction using short polypeptide clusters as features. Our study suggests that the short polypeptide cluster may help better understand the functionalities of proteins.
\end{abstract}

\section{Background}

Defining the functional relationships between proteins is essential to understand many aspects of biology. A classical approach of understanding gene functional relationships is to produce phenotype of combination mutant in two genes [1]; such relationships are called genetic interactions. Recently, high throughput methods [2-4] have been developed to generate large scale genetic interactions in model organisms, such as yeast [5], Schizosaccharomyces pombe [6] and E. coli. [7]. The large scale genetic interactions have attracted much attention as they are capable of defining the genomewide functional relationships among proteins and are fundamental to comprehensive understanding of the organization of biological systems $[5,8,9]$. However, even with high throughput methods [2-4], experimental

\footnotetext{
* Correspondence: luofeng@clemson.edu

${ }^{1}$ School of Computing, Clemson University, Clemson, SC 29634, USA

Full list of author information is available at the end of the article
}

mapping of genetic interactions is still extremely labor intensive and one cannot screen genome-wide combinations in multiple cell organisms with ten thousands of genes as of now [10]. Thus, it is imperative to develop computational approaches to predict genome-wide genetic interactions and help complement and enhance wet-lab studies.

In extreme cases, mutation of two nonessential genes can lead to lethal phenotype; this kind of genetic interaction is called synthetic lethal genetic interaction (SLGI). Figure 1 illustrates one such synthetic lethal genetic interaction. The SLGIs are of interest because they are able to reveal functional relationships between proteins, pathways and complexes [11-13]. Two synthetic lethal genes have high probability of occurrence in compensatory pathways [14] or compensatory complexes [15]. Furthermore, the SLGIs are potentially useful in finding drug targets or drug combinations [16].
C Biomed Central

C 2012 Zhang et al.; licensee BioMed Central Ltd. This is an open access article distributed under the terms of the Creative Commons Attribution License (http://creativecommons.org/licenses/by/2.0), which permits unrestricted use, distribution, and reproduction in any medium, provided the original work is properly cited. 


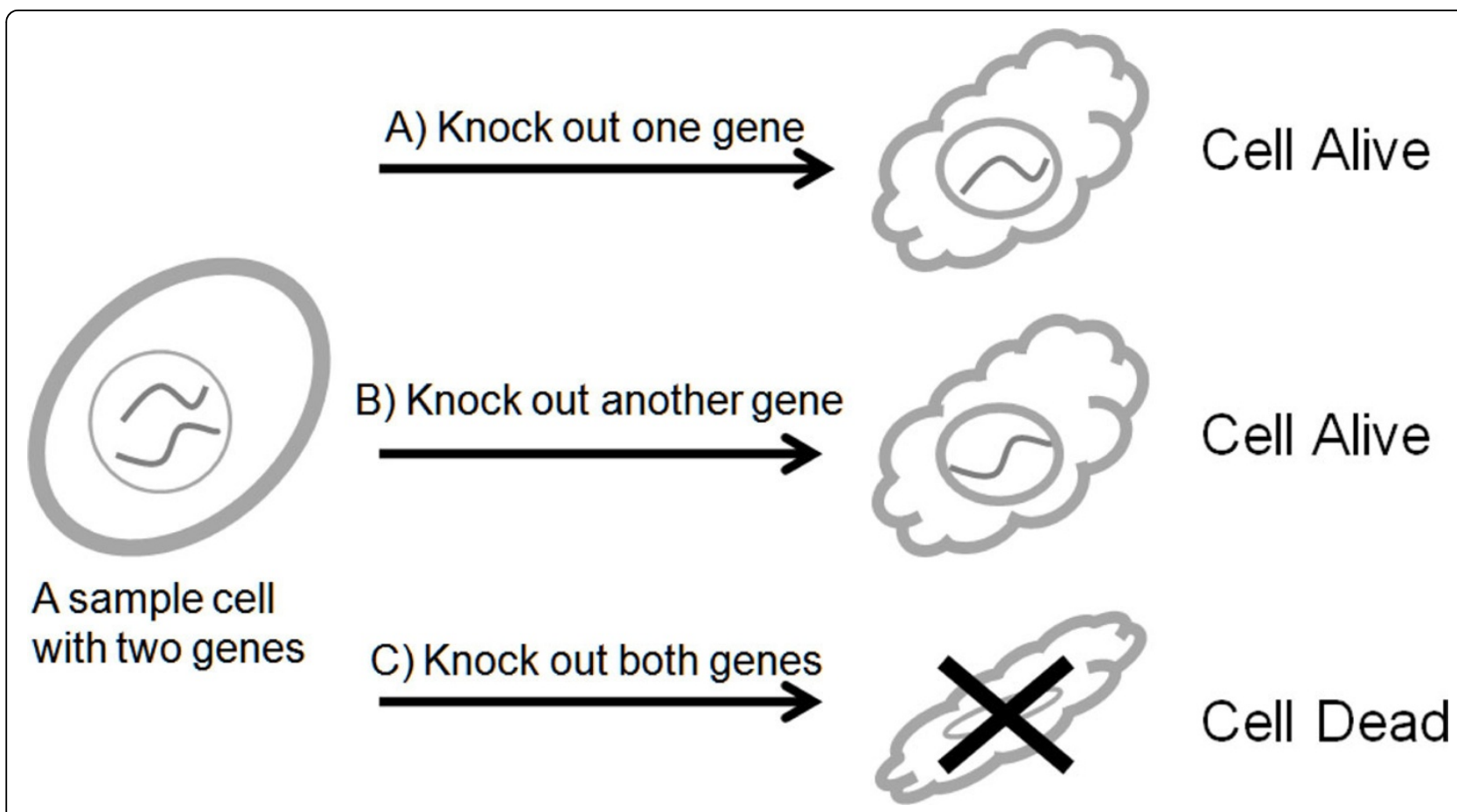

Figure 1 Illustration of synthetic lethal genetic interaction. A) and B) the cell is still alive after knocking out one gene; C) the cell died after knocking out both genes.

Prediction of SLGIs is impeded by the limit of understanding of genetic interactions. Unlike protein-protein interactions that are known as physical dockings among proteins, the molecular mechanism under genetic interactions has not been fully understood. Thus, it is difficult to select features and understand how features are related to SLGIs. Several computational approaches have been proposed for prediction of SLGIs, and many features, such as protein interactions, gene expression, functional annotation, gene location, protein network characteristics, and genetic phenotype, have been used by these approaches [10,17-20]. However, those methods depend on other genome-wide experimental results.

It is known as a virtual axiom in biology that the "sequence specifies structure and structure determines functionality" [21]. We hypothesize that it is possible to predict the SLGIs using the characteristics of protein sequence alone. Recently, we demonstrated that the yeast synthetic lethal genetic interactions can be explained by the genetic interactions between domains of those proteins [22]. Representing the structures and function of proteins, protein domains are usually regarded as building blocks of proteins and are conserved during evolution. Our studies showed that the domain genetic interactions are new type of relationship between protein domains. Moreover, we found that different domains in multi-domain yeast proteins contribute to their genetic interactions differently. The domain genetic interactions help define more precisely the function related to the synthetic lethal genetic interactions, and then help understand how domains contribute to different functionalities of multi-domain proteins. Using the probabilities of domain genetic interactions, we were able to predict novel yeast synthetic lethal genetic interactions.

However, the feasibility of domain based prediction is limited by the coverage of protein domains. For example, only 4480 of more than 6700 yeast proteins contain PfamA domains. In this study, we used the short polypeptide sequences, which are typically shorter than the classically defined protein domains, to characterize the functionalities of proteins. We demonstrated that the genetic interaction between a pair of proteins can be determined by the genetic interactions between the short polypeptide clusters of those proteins. Using short polypeptide clusters as features, we can not only increase the prediction coverage, but also improve the prediction performance.

\section{Results}

Identifying significant short polypeptide sequence pairs We constructed the short polypeptide clusters in three steps. First, we identified significant short polypeptide sequence pairs from yeast proteins based on the similarities of local alignments. For each yeast protein $A$, we chopped its protein sequence into short polypeptides 
with length of $L$ sequentially in a moving window size $w$. Then, we used the Smith-Waterman algorithm [23] to search all local matches with similarity scores beyond a predefined threshold for each short polypeptide of protein $A$ against the sequences of all other yeast proteins (Figure 2). We used BLOSUM62 to score the similarity. A significant local match between a short polypeptide sequence $a i$ from protein $A$ and a short polypeptide sequence $b j$ from protein $B$ indicates a polypeptide sequence pair $a i$ and $b j$. In addition, if using $b j$ from protein $B$ as query can find $a i$ from protein $A$ as a significant local match, the short polypeptide pair $a i$ and $b j$ is identified as a significant polypeptide sequence pair.

The significance values of local matching of short polypeptide sequences are determined using a p-value threshold. For each short polypeptide, We compared each short polypeptide to all other short polypeptide sequences and obtained a series of similarity scores. We then modeled those scores using an extreme value distribution. Based on a predefined p-value threshold, we determined the similarity score threshold for local matching. A local match is significant if its score beyond the similarity score threshold. For each short polypeptide sequence, with the same p-value, the similarity score threshold to determine the significant local matching are different.

We considered following parameters to experiment with our method: 1) size L of the short polypeptide sequence; 2) moving window size $w$; 3) penalty for gap and mismatch in the alignment; 4) p-value for the threshold of similarity score. In this study, we chose the size of each short polypeptide sequence $L$ to be 25 and the window $w$ was set to 5 . The penalty for gap and mismatch was chosen to be 14 . And, we have used pvalue equal to $10^{-6}$ for the threshold of similarities. We eventually obtained 3,353,962 short polypeptide sequence pairs covering 6711 yeast proteins. And there are totally 357,256 unique polypeptide sequences involved in these polypeptide sequence pairs.

\section{Clustering short polypeptide sequences}

After identifying significant polypeptide sequence pairs, we developed a clustering algorithm to group similar short polypeptide sequences into clusters. Initially, each significant polypeptide sequence pair was considered as a polypeptide sequence cluster. We first align the significant polypeptide sequence pair using ClustalW [24]. Then, we built a hidden Markov model (HMM) using the output of the multiple sequence alignment as the seed. The HMM model is constructed by the HMMbuild tool from HMMER [25]. After that, we searched the similar short polypeptide sequences using HMM model against all 357,256 short polypeptide sequences. The HMMsearch in HMMER [25] was used to screen similar polypeptide sequences with significances beyond a threshold. The similar short polypeptide sequences were added to the cluster. Then, the above process was repeated until no new short polypeptide sequence was added.

Several stringent thresholds $\left(10^{-10}, 10^{-15}\right.$ and $\left.10^{-20}\right)$ for HMMsearch were tested in order to include all similar short polypeptide sequences and reduce false cluster members. Due to the large size of the short polypeptide sequence pairs, we first obtained a cluster using each short polypeptide sequence pair as the seed. Then, we post-processed the short polypeptide sequence clusters. This strategy allowed us to easily run the clustering algorithm on a computer cluster.

\section{Post-processing short polypeptide clusters}

Our goal is to use the short polypeptide clusters to represent the functionalities of proteins, like the protein domains were used in [26]. First, we removed the duplicate short polypeptide clusters. Second, we merged two short polypeptide clusters together under 3 conditions: if the clusters share: 1) one polypeptide sequence; 2) $10 \%$ of polypeptide sequences of smaller cluster; and 3 ) $20 \%$ of polypeptide sequences of smaller cluster. Although we used a loose merging criterion, the

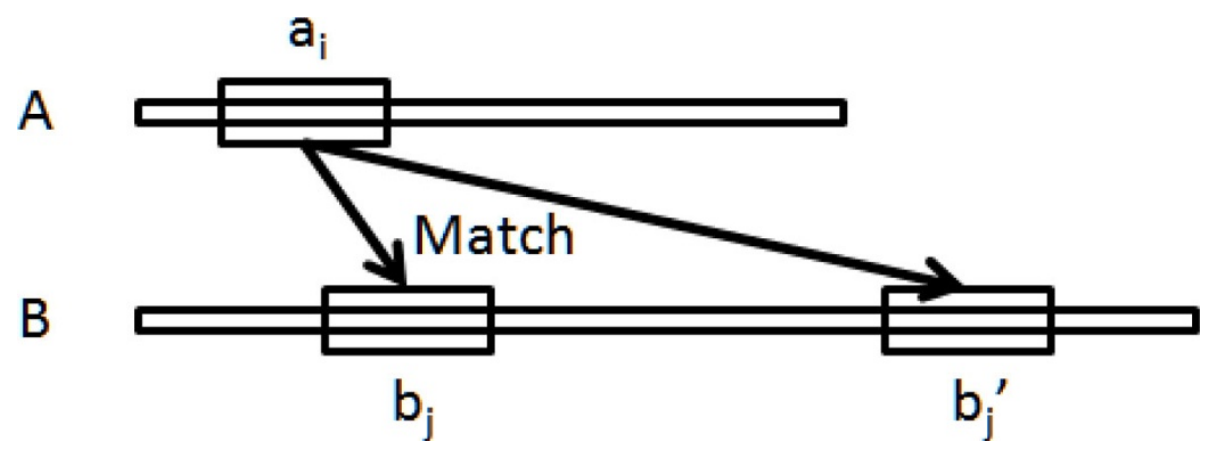

Figure 2 Illustration of matching between significant short polypeptide sequence pairs 
stringent thresholds used by HMMsearch still allowed the short polypeptides in each cluster to have high similarity. Figure 3 shows the multiple sequence alignment of short polypeptides in a merged cluster with 14 short polypeptides. We observed that those short polypeptide sequences in this cluster are highly conserved with significant number of identical amino acids.

Then, we filtered out the short polypeptide clusters with only two polypeptide sequences as those cluster will have no power to predict the SLGIs. We also filtered out short polypeptide clusters that existed in a large number of proteins. Those common short polypeptide clusters exist in both positive and negative data and also do not provide prediction power.

Next, we investigated how the choice of thresholds for HMMsearch and for filtering clusters affects the short polypeptide clusters. We tested different threshold configurations that combined one of three E-value thresholds for HMMsearch: $10^{-10}, 10^{-15}, 10^{-20}$; and one of four thresholds for filtering out short polypeptide clusters: 20, 50, 100, and No Filter. Table 1, 2 and 3 list the number of retrained short polypeptide clusters, the number of proteins and the number of SLGIs covered by the short polypeptide clusters, respectively. In three tables, the first column lists the thresholds for filtering out short polypeptide clusters; the first row lists the threshold for cluster merge and the second row lists the E-value thresholds used by HMMsearch. The results showed that increasing the cluster merge threshold will increase the number of covered proteins and number of short polypeptide clusters. Meanwhile, reducing the Evalue threshold from $10^{-10}$ to $10^{-15}$ also increases the number of covered proteins and number of short polypeptide clusters.
However, further reducing the E-value threshold to 10 ${ }^{-20}$ did not change the number of covered proteins and number of short polypeptide clusters.

The results showed that the short polypeptide clusters covered more proteins. For example, 5073 proteins are covered by the short polypeptide clusters obtained using $10^{-15}$ as HMMsearch threshold, $10 \%$ as cluster merge threshold and 50 as polypeptide cluster filtering threshold, comparing to 4480 proteins covered by PfamA domains. The maximum number of polypeptide clusters contained by a protein is 54 using this parameter configuration. The results indicate that the coverage of short polypeptide clusters in yeast proteins is higher than that using PfamA domains. The results also showed that the short polypeptide clusters covered similar number of SLGIs. For example, compared to 7702 SLGIs covered by PfamA domains, our polypeptide clusters cover 7681 SLGIs.

\section{Predicting yeast synthetic lethal genetic interactions using short polypeptide clusters by maximum likelihood estimation (MLE) approach}

In order to demonstrate the superiority of using short polypeptide clusters to predict SLGIs, we first obtained the probabilities of genetic interactions between short polypeptide clusters, and then used them to predict the probabilities of yeast SLGIs. We assumed that the genetic interaction between two short polypeptide clusters is independent and applied the Maximum Likelihood estimation (MLE) approach to estimate the probabilities of short polypeptide clusters.

We compared the MLE methods based on short polypeptide clusters, obtained using different HMMsearch, cluster filtering and cluster merge thresholds, to the

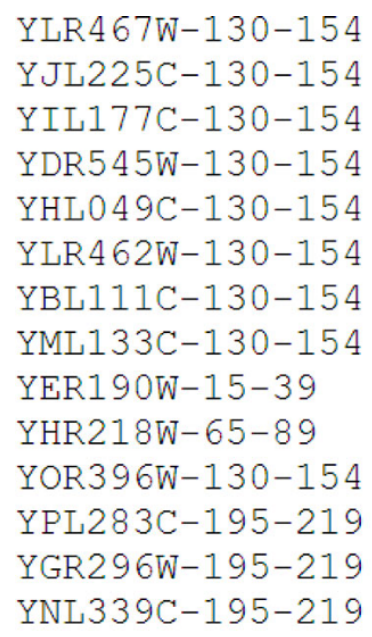

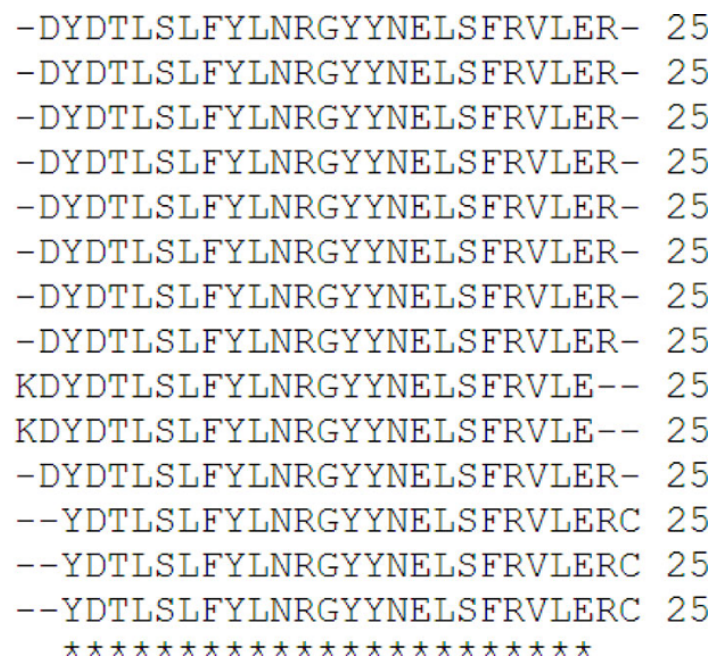

Figure 3 Multiple sequence alignment of a merged short polypeptide cluster. The stars in the bottom of the figure indicate the conserved identical amino acids. 
Table 1 The number of short polypeptide clusters obtained with various thresholds for HMMsearch, cluster filtering and cluster merge.

\begin{tabular}{clllllllll}
\hline Threshold & \multicolumn{3}{c}{ Merge with at least one overlap } & \multicolumn{3}{c}{ Merge with at least $\mathbf{1 0 \%}$ overlap } & \multicolumn{3}{c}{ Merge with at least 20\% overlap } \\
& $\mathbf{1 0}^{-\mathbf{1 0}}$ & $\mathbf{1 0}^{-\mathbf{1 5}}$ & $\mathbf{1 0}^{-\mathbf{2 0}}$ & $\mathbf{1 0}^{-\mathbf{1 0}}$ & $\mathbf{1 0}^{\mathbf{- 1 5}}$ & $\mathbf{1 0}^{-\mathbf{2 0}}$ & $\mathbf{1 0}^{-\mathbf{1 0}}$ & $\mathbf{1 0}^{\mathbf{- 1 5}}$ & $\mathbf{1 0}^{\mathbf{- 2 0}}$ \\
\hline $\mathbf{2 0}$ & 4188 & 5324 & 5326 & 4443 & 5807 & 5797 & 4760 & 6443 & 6399 \\
$\mathbf{5 0}$ & 4188 & 5330 & 5332 & 4471 & 5839 & 5822 & 4793 & 6475 & 6427 \\
$\mathbf{1 0 0}$ & 4188 & 5330 & 5332 & 4479 & 5848 & 5831 & 4803 & 6485 & 6432 \\
No Filter & 4189 & 5331 & 5333 & 4486 & 5856 & 5840 & 4812 & 6494 & 6440 \\
\hline
\end{tabular}

MLE method based on protein domains. We trained those MLE methods using all SLGIs covered by features. The MLE based on short polypeptide clusters were able to assign the probabilities of genetic interaction to more protein pairs. For example, the MLE method based on short polypeptide clusters using $10^{-15}$ as HMMsearch threshold, $10 \%$ as cluster merge threshold and 50 as polypeptide cluster filtering threshold were able to assign the probabilities of genetic interaction to $1,060,860$ protein pairs while the MLE based protein domains can only assign probabilities of genetic interaction to 536,175 protein pairs. This result showed that short polypeptide cluster based approach provides a much higher coverage to predict SLGIs.

To further evaluate the performance of short polypeptide cluster based MLE method, we tested the MLE methods on an experimentally obtained genetic interactions and non-genetic interactions, which include 3771 SLGIs and 688,045 non- SLGIs [10]. This data have been used by Wong et al. [10]. So we refer this experimental data as Wong data. We first trained the MLE method using SLGIs that are not included in the Wong data. Then, we assigned the probabilities of genetic interaction to the SLGIs and non SLGIs in Wong data. Table 4 lists the AUC (area under ROC curve) values for predicting Wong data of MLE methods based on short polypeptide clusters obtained using different HMMsearch, cluster merge and cluster filtering thresholds. The performance of MLE based on short polypeptide clusters is slightly better than that of MLE method based on protein domains. The AUC score for MLE based on short polypeptide clusters using $10^{-15}$ as HMMsearch threshold, $10 \%$ as cluster merge threshold and 50 as polypeptide cluster filtering threshold is
0.6761 while the AUC score for MLE based on protein domains is 0.6567 .

\section{Discussion and conclusions}

In this study, we developed a framework to identify significant short polypeptide clusters from yeast protein sequences. We hypothesized that those short polypeptide clusters represent the functionalities of proteins, like the protein domains. We then used these short polypeptide clusters as features to predict yeast synthetic lethal genetic interactions. The short polypeptide cluster based approach provides much higher coverage for predicting yeast synthetic lethal genetic interactions. Evaluation using experimental data sets showed that the short polypeptide cluster based approach can achieve higher performance than the previous protein domain based approach.

In future, we would like to continue improve the identification of short polypeptide clusters. Moreover, it is worthwhile to develop methods to understanding those short polypeptide clusters. Annotating those short polypeptide clusters may help better understand the functionalities of protein domains.

\section{Methods}

\section{Source of data}

We downloaded the yeast synthetic lethal genetic interactions from the Saccharomyces Genome Database (SGD) [27] (February 2011 version). There were totally 11011 synthetic lethal genetic interactions. We downloaded the protein sequences of yeast from GenBank [28]. There are totally 6717 proteins with sequences. The minimum and maximum lengths of the protein sequences in yeast are 16 and 4901 respectively. The

Table 2 The number of proteins covered with various thresholds for HMMsearch, cluster filtering, and cluster merge.

\begin{tabular}{|c|c|c|c|c|c|c|c|c|c|}
\hline \multirow[t]{2}{*}{ Threshold } & \multicolumn{3}{|c|}{ Merge with at least one overlap } & \multicolumn{3}{|c|}{ Merge with at least $10 \%$ overlap } & \multicolumn{3}{|c|}{ Merge with at least $20 \%$ overlap } \\
\hline & $10^{-10}$ & $10^{-15}$ & $10^{-20}$ & $10^{-10}$ & $10^{-15}$ & $10^{-20}$ & $10^{-10}$ & $10^{-15}$ & $10^{-20}$ \\
\hline 20 & 4262 & 4556 & 4561 & 4661 & 4911 & 4907 & 4840 & 5063 & 5061 \\
\hline 50 & 4262 & 4585 & 4590 & 4858 & 5073 & 5058 & 5050 & 5201 & 5216 \\
\hline 100 & 4262 & 4585 & 4590 & 4947 & 5176 & 5135 & 5150 & 5303 & 5253 \\
\hline No Filter & 5693 & 5630 & 5630 & 5693 & 5630 & 5630 & 5693 & 5630 & 5630 \\
\hline
\end{tabular}


Table 3 The number of SLGIs retained with various thresholds for HMMsearch, cluster filtering, and cluster merge.

\begin{tabular}{clllllllll}
\hline Threshold & \multicolumn{3}{c}{ Merge with at least one overlap } & \multicolumn{3}{c}{ Merge with at least $\mathbf{1 0 \%}$ overlap } & \multicolumn{3}{c}{ Merge with at least 20\% overlap } \\
& $\mathbf{1 0}^{-\mathbf{1 0}}$ & $\mathbf{1 0}^{-\mathbf{1 5}}$ & $\mathbf{1 0}^{-\mathbf{2 0}}$ & $\mathbf{1 0}^{-\mathbf{1 0}}$ & $\mathbf{1 0}^{\mathbf{- 1 5}}$ & $\mathbf{1 0}^{-\mathbf{2 0}}$ & $\mathbf{1 0}^{-\mathbf{1 0}}$ & $\mathbf{1 0}^{\mathbf{- 1 5}}$ & $\mathbf{1 0}^{\mathbf{- 2 0}}$ \\
\hline $\mathbf{2 0}$ & 6143 & 6648 & 6650 & 7110 & 7386 & 7408 & 7289 & 7820 & 7873 \\
$\mathbf{5 0}$ & 6143 & 6800 & 6802 & 7604 & 7681 & 7757 & 7770 & 8109 & 8370 \\
$\mathbf{1 0 0}$ & 6143 & 6800 & 6802 & 7710 & 7923 & 7951 & 8064 & 8376 & 8522 \\
No Filter & 9592 & 9427 & 9427 & 9592 & 9427 & 9427 & 9592 & 9427 & 9427 \\
\hline
\end{tabular}

average length is 450 and the standard deviation of the protein sequences is 380 .

\section{Determination of local alignment similarity score threshold}

The distribution of scores of local alignments between a short polypeptide and all other short polypeptide sequences can be described by extreme value distribution (EVD):

$$
F(x)=e^{-e^{\frac{x-\mu}{\beta}}}
$$

and the parameters of the EVD can be estimated by:

$$
\begin{aligned}
& \beta=\frac{\sigma \sqrt{6}}{\pi} \\
& \mu=\bar{X}-0.5772 \beta
\end{aligned}
$$

where $\bar{X}$ and $\sigma$ are the sample mean and standard deviation, respectively.

Based on Karlin-Altshcul statistics [29], the expected number of high-scoring segment pairs (HSPs) with score higher than $\mathrm{S}$ can be obtained by:

$$
E=K m n e^{-\lambda S}
$$

where $m$ and $n$ are the lengths of the two sequences being compared. The parameter $K$ and $\lambda$ can be obtained from parameters of the EVD:

$$
\begin{aligned}
& \lambda=1 / \beta \\
& K=e^{\frac{\mu}{\beta}} / m n
\end{aligned}
$$

The p-value of finding at least one HSP with score higher than $\mathrm{S}$ can be obtained by [29]:

$$
P=1-e^{-E}
$$

With a given p-value, we can get a corresponding $\mathrm{E}$ value. The parameter $K$ and $\lambda$ can be estimated by sample mean and standard deviation of scores. Significant similarity score can be computed by equation (4).

\section{Algorithm to cluster short polypeptide sequences}

The short polypeptide sequence clustering method implemented is summarized as follows:

Input: a pair of short polypeptide sequences

Initialization: add the short polypeptide sequence pair into cluster

Step 1. Conduct multiple sequence alignment (MSA) for the sequences in the cluster using ClustalW;

Step 2. Build a HMM model using HMMbuild from the output of MSA in step 1;

Step 3. Search all similar short polypeptide sequences using HMMsearch and add them to the cluster. If no new short polypeptide sequence is added, stop. Else, go back to step 1 .

\section{Estimation of probabilities and significances of domain} genetic interactions

We treated the protein SLGIs $L_{m, n}$ and short polypeptide cluster genetic interactions $C_{i, j}$ as random variables. $L_{m, n}=1$ if two proteins $i$ and $j$ genetically interact and $L_{m, n}=0$ otherwise. $C_{i, j}=1$ if two short polypeptide clusters $i$ and $j$ genetically interact and $C_{i, j}=0$ otherwise. We estimated the probabilities of potential short polypeptide

\begin{tabular}{|c|c|c|c|c|c|c|c|c|c|}
\hline \multirow[t]{2}{*}{ Threshold } & \multicolumn{3}{|c|}{ Merge with at least one overlap } & \multicolumn{3}{|c|}{ Merge with at least $10 \%$ overlap } & \multicolumn{3}{|c|}{ Merge with at least $20 \%$ overlap } \\
\hline & $10^{-10}$ & $10^{-15}$ & $10^{-20}$ & $10^{-10}$ & $10^{-15}$ & $10^{-20}$ & $10^{-10}$ & $10^{-15}$ & $10^{-20}$ \\
\hline 20 & 0.630 & 0.639 & 0.636 & 0.644 & 0.647 & 0.642 & 0.627 & 0.665 & 0.627 \\
\hline 50 & 0.630 & 0.638 & 0.634 & 0.669 & 0.676 & 0.644 & 0.662 & 0.671 & 0.633 \\
\hline 100 & 0.630 & 0.659 & 0.634 & 0.644 & 0.628 & 0.645 & 0.624 & 0.628 & 0.636 \\
\hline
\end{tabular}
cluster interactions $\operatorname{Pr}\left(C_{i, j}=1\right)$ by maximizing the likelihood of observed genetic interactions using the Expectation-Maximization (EM) algorithm [30-32]. The EM algorithm iteratively estimates the maximum likelihood

Table 4 The AUC values for the predictions of Wong data using different short polypeptide clusters obtained with various thresholds for HMMsearch cluster filters, and cluster merges. 
of the 'complete data' that combine the observed data and unobserved data. Here, the protein genetic interactions and the short polypeptide cluster information of proteins are our observed data and the short polypeptide cluster genetic interactions are our unobserved data.

Assuming short polypeptide cluster genetic interactions are independent, the likelihood of observed protein genetic interactions based on short polypeptide cluster genetic interactions can be obtained as:

$$
L=\prod_{i, j} \operatorname{Pr}\left(C_{i, j}=1\right)^{M_{i, j}+a}\left(1-\operatorname{Pr}\left(C_{i, j}=1\right)\right)^{N_{i, j}+K_{i, j}+b}
$$

where $M_{i, j}$ is the number of genetic interacting pairs between short polypeptide clusters $i$ and $j$ in all protein genetic interactions; $\mathrm{N}_{\mathrm{i}, \mathrm{j}}$ is the number of non genetic interacting short polypeptide cluster pairs between $\mathrm{i}$ and $j$ in protein genetic interactions; and $K_{i, j}$ is the number of non genetic interacting protein pairs including $i$ in one protein and $j$ in the other one. The value of $K_{i, j}$ is computed by counting all possible protein pairs with $\mathrm{i}$ in one protein and $j$ in the other one with excluding the known genetic interacting protein pairs. The $\mathrm{K}_{\mathrm{i}, \mathrm{j}}$ will remain unchanged during EM computation. The constants $a$ and $b$ are pseudo counts to avoid the $\operatorname{Pr}\left(\mathrm{C}_{\mathrm{i}, \mathrm{j}}=1\right)$ or $\operatorname{Pr}\left(\mathrm{C}_{\mathrm{i}, \mathrm{j}}=0\right)$ to be zero when instances of domains $\mathrm{i}$ and $\mathrm{j}$ are rare. We set both $a$ and $b$ to 1 in our calculation.

Initially, $\mathrm{M}_{\mathrm{i}, \mathrm{j}}$ was set to the number of genetic interactions between domain $i$ and $j$ in experimental genetic interactions; $N_{i, j}$ is set to 0 . And $\operatorname{Pr}\left(C_{i, j}=1\right)$ was initialized as following:

$$
\operatorname{Pr}\left(\mathrm{C}_{\mathrm{i}, \mathrm{j}}=1\right)=\frac{\mathrm{M}_{\mathrm{i}, \mathrm{j}}}{\mathrm{M}_{\mathrm{i}, \mathrm{j}}+N_{i, j}+K_{i, j}}
$$

In each Expectation step of EM algorithm, we first estimated the expected values of $E\left[\mathrm{M}_{\mathrm{i}, j}\right]$ and $\mathrm{E}\left[\mathrm{N}_{\mathrm{i}, \mathrm{j}}\right][31]$ using the current $\operatorname{Pr}\left(C_{i, j}=1\right)$ :

$$
\begin{aligned}
& E\left[M_{i, j}\right]=\sum_{m, n}\left[\frac{\operatorname{Pr}\left(C_{i, j}=1\right)}{1-\Pi_{i \subset C(m), j \subset C(n)}\left(1-\operatorname{Pr}\left(C_{i, j}=1\right)\right)}\right] \\
& E\left[N_{i, j}\right]=\sum_{m, n}\left(1-\left[\frac{\operatorname{Pr}\left(C_{i, j}=1\right)}{1-\Pi_{i \subset C(m), j \subset C(n)}\left(1-\operatorname{Pr}\left(C_{i, j}=1\right)\right)}\right]\right)
\end{aligned}
$$

Then, we calculate the $\operatorname{Pr}\left(\mathrm{C}_{\mathrm{i}, \mathrm{j}}=1\right)$ using the $\mathrm{E}\left[\mathrm{M}_{\mathrm{i}, \mathrm{j}}\right]$ and $\mathrm{E}\left[\mathrm{N}_{\mathrm{i}, \mathrm{j}}\right]$ as following (Maximization step):

$$
\operatorname{Pr}\left(C_{i, j}=1\right)=\frac{E\left[M_{i, j}\right]+a}{E\left[M_{i, j}\right]+E\left[N_{i, j}\right]+K_{i, j}+a+b}
$$

The EM algorithm was over the Expectation and Maximization steps until the change of likelihood $L$ is less than a pre-defined small value.
We assumed that two proteins genetically interact $\left(L_{m}\right.$, ${ }_{n}=1$ ) if and only if at least one domain pair from the two proteins genetically interact $\left(C_{i, j=1}\right)$. Then, we calculated the probability of two proteins genetically interacting $\operatorname{Pr}\left(L_{m, n}=1\right)$ as following:

$$
\operatorname{Pr}\left(L_{m, n}=1\right)=1.0-\prod_{\substack{i \in C(m) \\ j \in C(n)}}\left(1-\operatorname{Pr}\left(C_{i, j}=1\right)\right)
$$

A pair of proteins was predicted to be SLGI only if its probability is higher than a predefined threshold.

\section{Acknowledgements}

This study was supported in part by the Institute for Modeling and Simulation Applications at Clemson University and by NSF Contracts DBI0960586 and CCF-0832582

This article has been published as part of Proteome Science Volume 10 Supplement 1, 2012: Selected articles from the IEEE International Conference on Bioinformatics and Biomedicine 2011: Proteome Science. The full contents of the supplement are available online at http://www.proteomesci. com/supplements/10/S1

\section{Author details}

${ }^{1}$ School of Computing, Clemson University, Clemson, SC 29634, USA. ${ }^{2}$ Electrical Engineering and Computer Science Department, University of Kansas, Lawrence, KS 66045-7621, USA.

\section{Authors' contributions}

FL and PKS designed research. BL and YHZ implemented the idea. FL, YHZ and XWC analyzed the results. All authors have read and approved the final manuscript.

\section{Competing interests}

The authors declare that they have no competing interests.

Published: 21 June 2012

\section{References}

1. Hartman J, Garvik B, Hartwell L: Principles for the buffering of genetic variation. Science 2001, 291:1001-1004.

2. Tong $A H$, Evangelista $M$, Parsons $A B, X u H$, Bader GD, Page N, Robinson $M$ Raghibizadeh S, Hogue CW, Bussey $\mathrm{H}$, et al: Systematic genetic analysis with ordered arrays of yeast deletion mutants. Science 2001, 294(5550):2364-2368

3. Pan X, Yuan DS, Ooi SL, Wang X, Sookhai-Mahadeo S, Meluh P, Boeke JD: dSLAM analysis of genome-wide genetic interactions in Saccharomyces cerevisiae. Methods 2007, 41(2):206-221.

4. Schuldiner M, Collins SR, Weissman JS, Krogan NJ: Quantitative genetic analysis in Saccharomyces cerevisiae using epistatic miniarray profiles (E-MAPs) and its application to chromatin functions. Methods 2006, 40(4):344-352.

5. Tong AHY, Lesage $G$, Bader GD, Ding $H$, Xu H, Xin X, Young J, Berriz GF, Brost $R L$, Chang $M$, et al: Global mapping of the yeast genetic interaction network. Science 2004, 303(5659):808-813

6. Roguev A, Wiren M, Weissman JS, Krogan NJ: High-throughput genetic interaction mapping in the fission yeast Schizosaccharomyces pombe. Nat Methods 2007, 4(10):861-866.

7. Butland G, Babu M, Diaz-Mejia JJ, Bohdana F, Phanse S, Gold B, Yang W, Li J, Gagarinova AG, Pogoutse O, et al: eSGA: E. coli synthetic genetic array analysis. Nat Methods 2008, 5(9):789-795.

8. Boone C, Bussey $\mathrm{H}$, Andrews BJ: Exploring genetic interactions and networks with yeast. Nature Reviews Genetics 2007, 8(6):437-449.

9. Winzeler EA, Shoemaker DD, Astromoff A, Liang $H$, Anderson $K$, Andre $B$, Bangham R, Benito R, Boeke JD, Bussey $H$, et al: Functional characterization of the $\mathrm{S}$. cerevisiae genome by gene deletion and parallel analysis. Science 1999, 285(5429):901-906. 
10. Wong SL, Zhang LV, Tong AHY, Li Z, Goldberg DS, King OD, Lesage G, Vidal M, Andrews B, Bussey H: Combining biological networks to predict genetic interactions. Proc Natl Acad Sci U S A 2004, 101(44):15682-15687.

11. Mani R, St Onge RP, Hartman JL, Giaever G, Roth FP: Defining genetic interaction. Proc Natl Acad Sci U S A 2008, 105(9):3461-3461.

12. Ye P, Peyser BD, Pan X, Boeke JD, Spencer FA, Bader JS: Gene function prediction from congruent synthetic lethal interactions in yeast. Mol Syst Biol 2005, 1:2005.0026.

13. Wong SL, Zhang LV, Roth FP: Discovering functional relationships: biochemistry versus genetics. Trends Genet 2005, 21(8):424-427.

14. Kelley R, Ideker T: Systematic interpretation of genetic interactions using protein networks. Nature biotechnology 2005, 23(5):561-566.

15. Le Meur N, Gentleman R: Modeling synthetic lethality. Genome Biology 2008, 9(9):R135.

16. Kaelin WG Jr: The concept of synthetic lethality in the context of anticancer therapy. Nat Rev Cancer 2005, 5(9):689-698.

17. Zhong W, Sternberg PW: Genome-wide prediction of C. elegans genetic interactions. Science 2006, 311:1481-1484.

18. Paladugu S, Zhao S, Ray A, Raval A: Mining protein networks for synthetic genetic interactions. BMC Bioinformatics 2008, 9(1):426-426.

19. Chipman KC, Singh AK: Predicting genetic interactions with random walks on biological networks. BMC Bioinformatics 2009, 10:17.

20. Ulitsky I, Krogan NJ, Shamir R: Towards accurate imputation of quantitative genetic interactions. Genome Biol 2009, 10(12):R140.

21. Anfinsen CB: Principles that Govern the Folding of Protein Chains. Science 1973, 181(4096):223-230

22. Li B, Cao W. Zhou J, Luo F: Understanding and predicting synthetic lethal genetic interactions in Saccharomyces cerevisiae using domain genetic interactions. BMC Systems Biology 2011, 5(1):73.

23. Smith TF, Waterman MS: Identification of common molecular subsequences. J Mol Biol 1981, 147(1):195-197.

24. Thompson JD, Gibson TJ, Higgins DG: Multiple sequence alignment using ClustalW and ClustalX. Curr Protoc Bioinformatics 2002, Chapter 2:Unit 2.3.

25. Eddy SR: Profile hidden Markov models. Bioinformatics 1998, 14(9):755-763.

26. Bateman A, Coin L, Durbin R, Finn RD, Hollich V, Griffiths Jones S, Khanna A, Marshall M, Moxon S, Sonnhammer ELL: The Pfam protein families database. Nucleic Acids Research 2004, 32(suppl 1):D138.

27. Cherry JM, Adler C, Ball C, Chervitz SA, Dwight SS, Hester ET, Jia Y, Juvik G, Roe T, Schroeder M: SGD: Saccharomyces genome database. Nucleic Acids Research 1998, 26(1):73-73.

28. Benson DA, Karsch-Mizrachi I, Lipman DJ, Ostell J, Wheeler DL: GenBank. Nucleic Acids Res 2005, 33:D34-38.

29. Karlin S, Altschul SF: Methods for assessing the statistical significance of molecular sequence features by using general scoring schemes. Proc Natl Acad Sci U S A 1990, 87(6):2264.

30. Deng M, Mehta S, Sun F, Chen T: Inferring domain-domain interactions from protein-protein interactions. Proceedings of the Sixth Annual International Conference on Computational Biology Washington, DC, USA. ACM; 2002, 117-126

31. Riley R, Lee C, Sabatti C, Eisenberg D: Inferring protein domain interactions from databases of interacting proteins. Genome Biology 2005, 6(10):R89-R89.

32. Dempster AP, Laird NM, Rubin DB: Maximum likelihood from incomplete data via the EM algorithm. Journal of the Royal Statistical Society Series $B$ (Methodological) 1977, 39(1):1-38.

\section{Submit your next manuscript to BioMed Central and take full advantage of:}

- Convenient online submission

- Thorough peer review

- No space constraints or color figure charges

- Immediate publication on acceptance

- Inclusion in PubMed, CAS, Scopus and Google Scholar

- Research which is freely available for redistribution

Submit your manuscript at www.biomedcentral.com/submit 